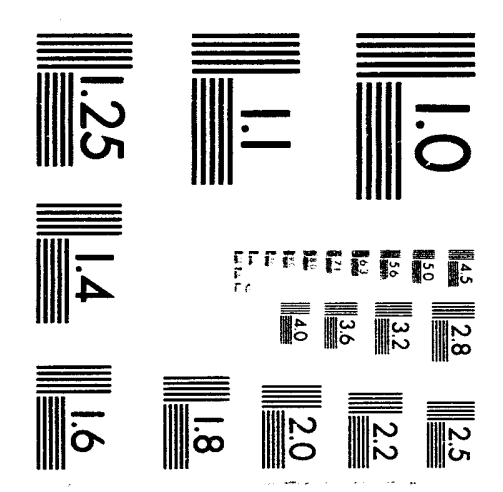


$\mathrm{BNL}-60214$

AD/RHIC-128

Informal Report

\title{
Tune Modulation Due to Synchrotron Oscillations and Chromaticity, and the Dynamic Aperture
}

\author{
George Parzen
}

\author{
March 1994
}

DISCLAIMER

\begin{abstract}
This report was prepared as an account of work sponsored by an agency of the United States Government. Neither the United States Government nor any agency thereof, nor any of their employees, makes any warranty, express or implied, or assumes any legal liability or responsibility for the accuracy, completeness, or usefulness of any information, apparatus, product, or process disclosed, or represents that its use would not infringe privately owned rights. Reference herein to any specific commercial product, process, or service by trade name, trademark, manufacturer, or otherwise does not necessarily constitute or imply its endorsement, recommendation, or favoring by the United States Government or any agency thereof. The views and opinions of authors expressed herein do not necessarily state or reflect those of the United States Government or any agency thereof.
\end{abstract}

\section{R H I C P R O J E C T}

\author{
Brookhaven National Laboratory \\ Associated Universities, Inc. \\ Upton, NY 11973
}

Under Contract No. DE-AC02-76CH00016 with the UNITED STATES DEPARTMENT OF ENERGY 


\section{Tune Modulation due to Synchrotron Oscillations and Chromaticity, and the Dynamic Aperture \\ George Parzen \\ March 1, 1994 \\ RECEIVED \\ APR 221994 \\ OSTI}

\section{Introduction}

A tracking study was done of the effects of a tune modulation, due to synchrotron oscillations and the tune dependence on momentum (chromaticity), on the dynamic aperture. The studies were done using several RHIC lattices and tracking runs of about $1 \times 10^{6}$ turns. The dynamic aperture was found to decrease roughly linearly with the amplitude of the tune modulation and may be represented by

$$
A=A_{0}(1-10 \Delta \nu)
$$

where $A_{0}$ is the dynamic aperture for $\Delta \nu=0$, and $\Delta \nu$ is the tune modulation amplitude. In Eq. (1), the range of $\Delta \nu$ is such that lower order non-linear resonances, like the 1/3 and $1 / 4$ resonance are not crossed because of the tume modulation.

Three different cases were studied, corresponding to RHIC lattices with different $\beta^{*}$, and with different synchrotron oscillation amplitudes. In each case, the tune modulation amplitude was varied by changing the chromaticity. In each case, roughly the same result, Eq. (1), was found.

The result found here for the effect of a tune modulation due to chromaticity may be compared with the result found ${ }^{1}$ for the effect of a tune modulation due to a gradient ripple in the quadrupoles, which was

$$
A=A_{0}(1-42 \Delta \nu)
$$

The effect of a $\Delta \nu$ due to a gradient ripple appears to be about 4 times stronger than the effect of a $\Delta \nu$ due to chromaticity and synchrotron oscillations.

\section{Tracking Results}

The ture of the particle is modulated with time due to the chromaticity and the synchrotron oscillations. If the chromaticity is given by $C_{x}, C_{y}$ and the amplitude of the synchrotron oscillation in momentum is given by $\pm \Delta p / p$, then the tune is modulated with time with a tune oscillation amplitude given by

$$
\begin{aligned}
\Delta \nu_{x} & =C_{x} \Delta p / p \\
\Delta \nu_{y} & =C_{y} \Delta p / p
\end{aligned}
$$


The frequency of the tune modulation is that of the synchrotron oscillation. For one case that was studied, the synchrotron oscillation frequency is about $260 \mathrm{hz}$.

Figure 1 shows the dynamic aperture $A_{S L}$ versus $\Delta \nu$ for a RHIC lattice with $\beta^{*}=6$ at all 6 insertions. The synchrotron oscillation amplitude is held constant at $\Delta p / p=0.005$, $\Delta \nu$ is varied by varying the chromaticity $C_{x}=C_{y}$ from 0 to 10 producing a rnaximum $\Delta \nu$ of $50 \times 10^{-3}$. The nominal tune is $\nu_{x}=28.826, \nu_{y}=28.821$ and with this range of $\Delta \nu$ low order resonances like the $1 / 3$ or $1 / 4$ resonances, are not crossed. Figure 1 shows a roughly linear decrease of the dynamic aperture with $\Delta \nu$ from $A_{S L}=9.5 \mathrm{~mm}$ at $\Delta \nu=0$ to $A_{S L}=6.5 \mathrm{~mm}$ at $\Delta \nu=50 \times 10^{-3}$.

ASL vS $\triangle v$

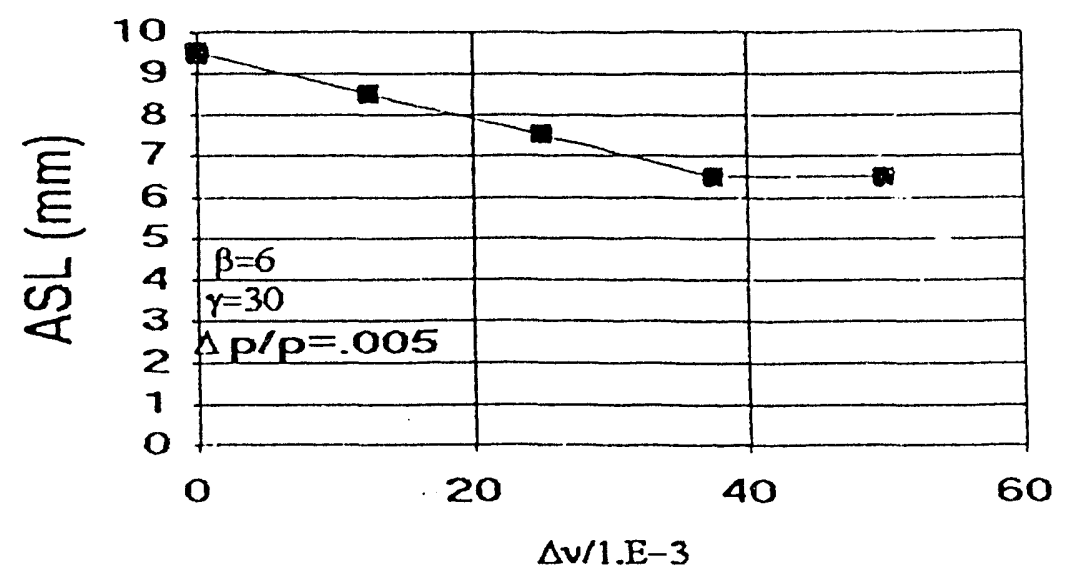

Figure 1: A plot of the dynamic aperture, $A_{S L}$, versus the tune modulation amplitude, $\Delta \nu$.

Three cases were studied with the same RF system which at the energy corresponding to $\gamma=30$ has a synchrotron oscillation frequency of about $260 \mathrm{hz}$. These three cases are listed in Table 1. Case 2 has $\beta^{*}=6$ and $\Delta p / p=0.0025$. Case 3 has $\beta^{*}=2$ and $\Delta p / p=0.0018$. The height of the RF bucket in $\Delta p / p$ is $\Delta_{B}=0.006$ in the 3 cases at $\gamma=30$. 
Table 1: A table of 3 cases studied where the synchrotron oscillation frequency was held constant at $f_{s}=260 \mathrm{~Hz}$.

\begin{tabular}{|c|c|c|}
\hline Case & $\beta^{*}$ & $\Delta p / p$ \\
& $(\mathrm{~m})$ & Synch. Osc. Amp. \\
\hline 1 & 6 & 0.005 \\
2 & 6 & 0.0025 \\
3 & 2 & 0.0018 \\
\hline
\end{tabular}

Figure 2 plots the tracking results for $A / A_{0}$ against $\Delta \nu$ for the 3 cases. $A$ is the dynamic aperture, $A_{0}$ is the dynamic aperture for $\Delta \nu=0$, and $\Delta \nu$ is the amplitude of the tune modulation, which is varied by changing the chromaticity $C_{x}=C_{y}$. Figure 2 indicates that the data for all 3 cases lie roughly on the straight line

$$
A=A_{0}(1-10 \Delta \nu)
$$

\section{A AO vs $\Delta v$}

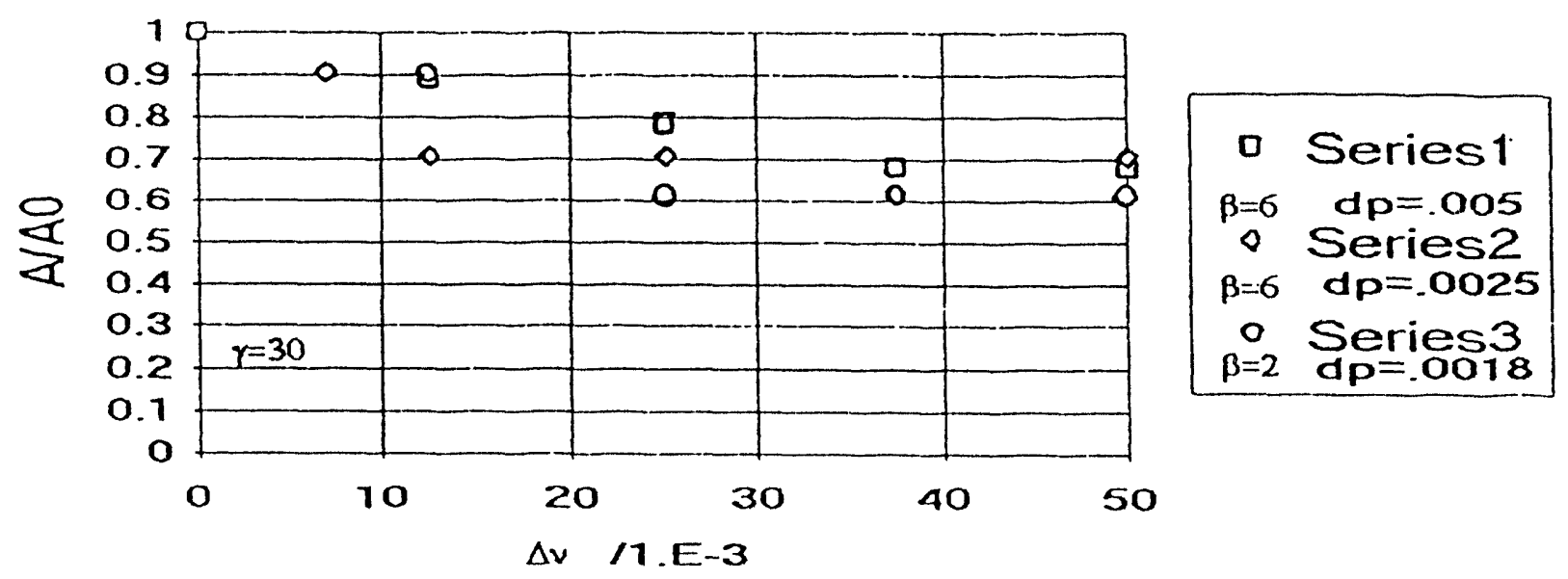

Figure 2: A plot of $A / A_{0}$ versus $\Delta y$ for three different cases. $A$ is the dynamic aperture, $A_{0}$ is the dynamic apertue for $\Delta \nu=0$.

In the range of $\Delta y$ cover in Fig. 2 there is only one low order resonance, which is below tenth order, that is reached by the tune modulation. This is the $5 / 6$ resonance at $\nu=28.83333$. The umperturbed tune is at $\nu_{x}=28.826 \nu_{y}=28.821$. The $5 / 6$ resonance is reached at a tune modulation of $\Delta \nu \geq 7 \times 10^{-3}$. The results in Fig. 2 do not clearly show the presence of this resonance. The runs done with $\Delta y \geq 7 \times 10^{-3}$ sweep over the 
$5 / 6$ resonance many times in $10^{6}$ turns, yet the dynamic aperture found for $\Delta \nu \geq 7 \times 10^{-3}$ does not show much effect from the $5 / 6$ resonance.

One may speculate as to under what conditions the result, Eq. (4) may be roughly valid. One may conjecture that Eq. (4) for the dependence of the dynamic aperture on the amplitude of the tune modulation may be roughly valid under the following conditions:

1. The tune modulation does not sweep over the lower order resonances like the $1 / 3$ or $1 / 4$ resonances.

2. The field error multipoles, $b_{n}$ or $a_{n}$, are roughly given by $b_{0} / R^{n}$, where $b_{0} \simeq 2 \times 10^{-4}$ and, usually, $R$ is roughly the magnet coil radius.

3. The tune modulation is generated by the presence of a tune dependence on momentum (chromaticity) and synchrotron (scillations, and the frequency of the synchrotron oscillations is small compared to the particle revolution frequency in the accelerator.

The result found for the decrease in dynamic aperture, Eq. (4), may be compared with the result found for the effect on the dynamic aperture due to a tune modulation generated by a ripple in the gradient of the quadrupoles, which is given by

$$
A=A_{0}(1-42 \Delta \nu), \text { gradient ripple. }
$$

One sees that a $\Delta y$ due a gradient ripple is more effective in reducing the dynamic aperture than a $\Delta \nu$ due to chromaticity and synchrotron oscillation by about a factor of 4 .

\section{Dependence of the Dynamic Aperture on the Synchrotron Oscillation Frequency}

The frequency of the synchrotron oscillation frequency can be varied by varying the voltage, $V$, and the harmonic number, $h$, of the RF cavity. If this is done holding $V / h$ constant, then the height of the RF bucket in $\Delta p / p$ is not changed.

The dependence of the dynamic aperture on the synchrotron oscillation frequency $f_{s}$ was studied for the case $\Delta \nu=50 \times 10^{-3}, \Delta p / p=0.005, C_{x}=C_{y}=10, \beta^{*}=6$. This case has the largest tune oscillation amplitude studied. The results for the dynamic aperture $A_{S L}$ vs. $f_{s}$ for this case are shown in Figure 3. $f_{s}$ was varied from $f_{s}=16.25 \mathrm{hz}$ to 520 hz. Figure 3 does not show much dependence of $A_{S L}$ on $f_{s}$ over this range in $f_{s} . A_{S L}$ is given by $5.5 \pm 1 \mathrm{~mm}$ over the range in $f_{s}$. There appears to be a small decrease in $A_{S L}$ for lower $f_{s}$. One should note that the tracking results for $A_{S L}$ become more doubtful at lower values $f_{s}$. At $f_{s}=16.25$, there is time for about 250 synchrotron periods in 1 million turns. This effect would probably reduce the dynamic aperture at lower values of $f_{s}$. 


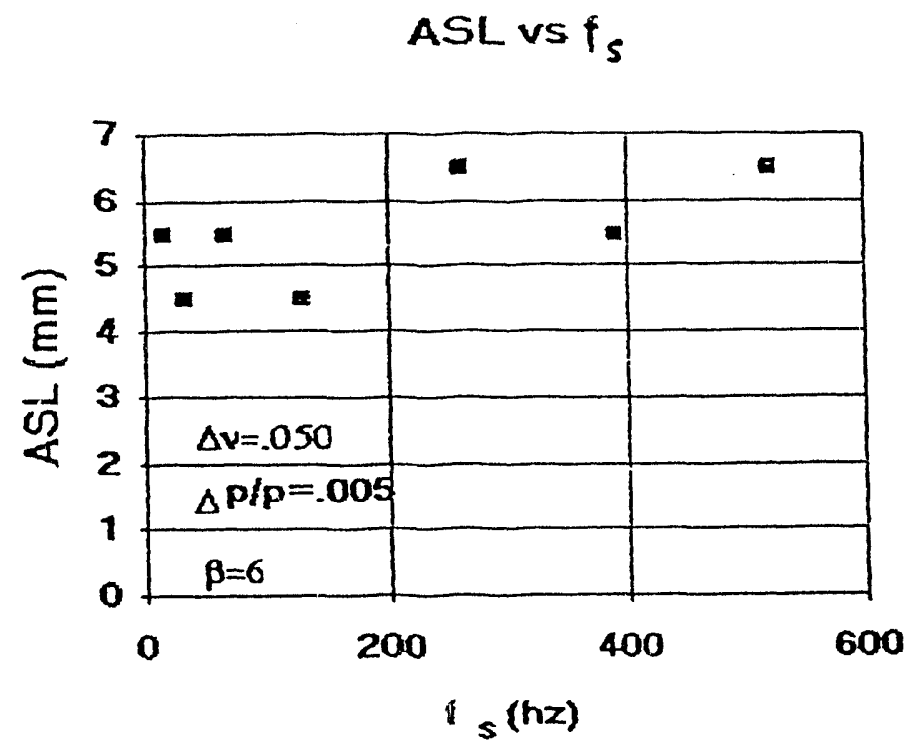

Figure 3: A plot of $A_{S L}$ versus $f_{s}$, the synchrotron oscillation frequency.

\section{Comments on the Tracking}

It is important that the tracking be symplectic. To achieve this, the ORBIT program was changed ${ }^{2}$ to allow the use of point magnets. The methods used are similar to those used in the TEAPOT ${ }^{3}$ program, with some modifications, including the choice of the reference orbit ${ }^{4}$.

One $160 \mathrm{MHz} R F$ cavity with an $\mathrm{RF}$ voltage of $4.5 \mathrm{MV}$ was used in the tracking. The bucket height is $\Delta p / p=6 \times 10^{-3}$ at $\gamma=30$ and $\Delta p / p=2 \times 10^{-3}$ at $\gamma=100$.

Random and systematic field errors were present in each magnet at the level given in Ref. 5. Field error multipoles up to order 10 were included. The studies done in this paper were done for a particular set of field errors which gave the smallest dynamic aperture, out of ten different distributions of the random field errors, in the absence of tune modulation.

The dynamic aperture is computed by doing a series of runs with the starting conditions $\epsilon_{x}=\epsilon_{y}, x^{\prime}=y^{\prime}=0$, and finding the largest betatron oscillation amplitude that is stable for 800,000 turns. 


\section{References}

1. G. Parzen, Tune Modulation due to Gradient Ripple and the Dynamic Aperture, BNL Report AD/RHIC-124 (1993).

2. G. Parzen, Effect of Synchrobetatron Coupling on the Dynamic Aperture, BNL Report AD/RHIC-119 (1993).

3. L. Schachinger and R. Talman, TEAPOT, A Thin Element Tracking Program, SSC Report SSC-52 (1985).

4. G. Parzen, Symplectic Tracking Using Point Magnets and a Reference Orbit Made of Circular Arcs and Straight Lines, BNL Report AD/RHIC-120 (1993).

5. RHIC Conceptual Design, BNL Report BNL-52195 (1989). 

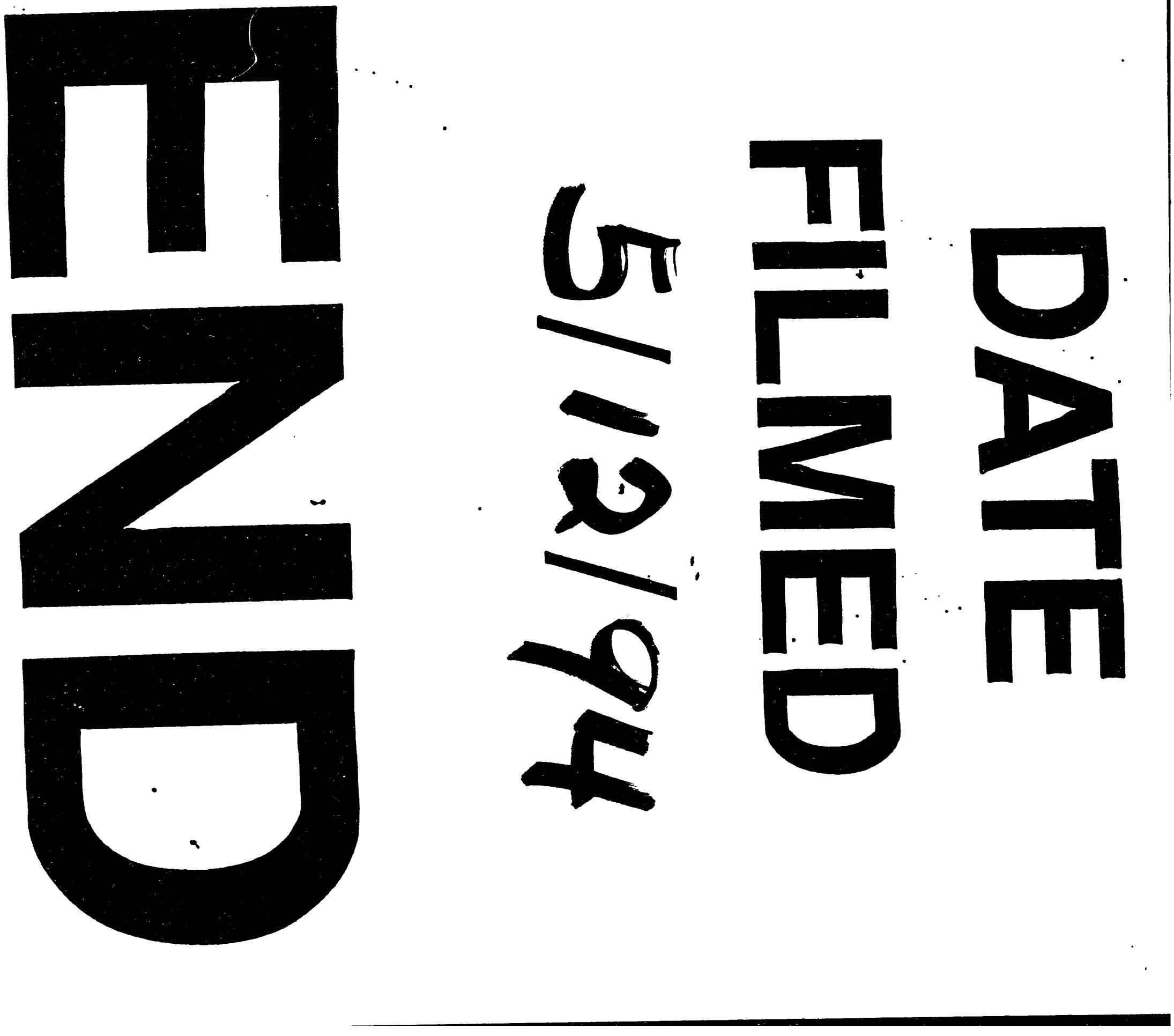

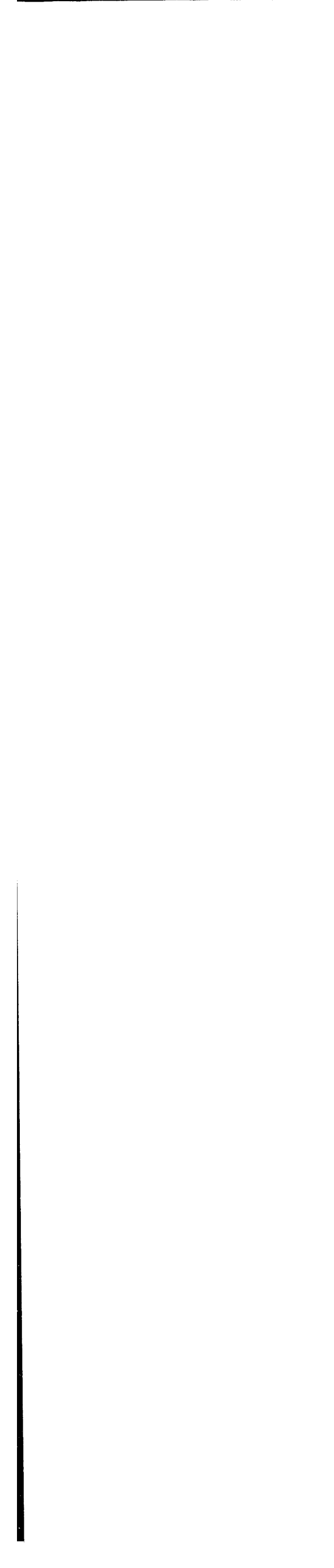$$
-
$$ 